\title{
ESTUDOS DAS CHUVAS MÁXIMAS PARA O MUNICÍPIO DE IGUATU-CE
}

\author{
Samara Alves de Souza ${ }^{1}$, Maria Holanda Coelho ${ }^{1}$, \\ Wedman de Lavor Vieira ${ }^{1}$, Adail Pinto CARdoso ${ }^{1}$, \\ Luiz Carlos Alves Bezerra ${ }^{1}$, Jỗo Coelho lima Verde Neto ${ }^{2}$ \\ ${ }^{1}$ Instituto Federal de Educação, Ciência e Tecnologia do Ceará (IFCE) - Campus Iguatu \\ ${ }^{2}$ Universidade Federal do Ceará (UFC) \\ <sawrah10@gmail.com>, <mehcoelho@yahoo.com.br>

$<$ wedmanifce@hotmail.com> <adailpintoo@yahoo.com.br> \\ <luisiguatu@hotmail.com>, <jclvneto@ hotmail.com>
}

DOI:10.21439/conexoes.v10i2.758

\begin{abstract}
Resumo. Informações sobre chuvas máximas prováveis são de grande importância no dimensionamento de projetos de engenharia hidráulica, drenagem urbana e rural, em perímetros de irrigação. Nesse contexto, este trabalho teve o objetivo de estimar as chuvas máximas para a cidade de Iguatu-CE para os períodos de retorno de 2, 5, 10, 20, 50 e 100 anos, através da distribuição de Gumbel. Para encontrar os parâmetros de Gumbel foi utilizado o método dos momentos. Foram utilizados dados de precipitação pluviométrica máxima diária anual e máxima diária mensal anual, expressos em altura de lâmina d'água (mm) de uma série de 38 anos. A precipitação máxima variou de $45,60 \mathrm{~mm} \mathrm{dia}^{-1}$ a $398,84 \mathrm{~mm}$ $\mathrm{dia}^{-1}$ para os diferentes períodos de retorno e os meses de novembro e dezembro são os que apresentaram menores e maiores valores de chuvas máximas respectivamente. O método de Gumbel mostrou-se adequado na estimativa das chuvas máximas para a cidade de Iguatu, o que representa uma alternativa para um planejamento de obras hidráulicas e de irrigação, minimizando assim os riscos de ocorrência de perdas econômicas.
\end{abstract}

Palavras-chaves: Precipitações extremas, Período de retorno, Método de Gumbel.

Abstract. Knowledge about probable maximum rainfall has a great importance to hydraulic engineering projects, as also rural, urban drainage and irrigation projects too. In this context, this study aimed to estimate the maximum rainfall for Iguatu-CE city for the return periods of 2, 5, 10, 20, 50 and 100 years, using the Gumbel distribution. To find the parameters of the Gumbel, it was used the moments method. I was employed the data collected about the daily maximum rainfall in a year and daily maximum in a month expressed in height of water blade $(\mathrm{mm})$ of a series of 38 years. The maximum rainfall was between 45,60 mm. dia ${ }^{-1}$ and $398,84 \mathrm{~mm} \cdot \mathrm{dia}^{-1}$ for the different return periods under study. The months of November and December presented the lowest and highest values of maximum rainfall respectively. The Gumbel method was suitable to estimate the maximum rainfall for the city of Iguatu, which represents an alternative to planning a hydraulic works and irrigation, thus minimizing the risks of economic losses.

Keywords: Extreme rainfalls, return periods, Gumbel method.

\section{INTRODUÇÃO}

A água é responsável por grande parte do desenvolvimento agrário de regiões onde os recursos hídricos são escassos, sendo a chuva a forma mais econômica e ambientalmente adequada de uso da água na agricultura

\section{(VIEIRA et al., 2010).}

A variabilidade das chuvas é um importante fator no controle do ciclo hidrológico e uma das variáveis climáticas que mais influência na transformação da paisagem e do meio ambiente (MARCUZZO; GOULARTE, 2012). A caracterização da variabilidade temporal das 
chuvas intensas é imprescindível para quantificar adequadamente os efeitos ocasionados, de modo especial, ao controle do escoamento superficial em áreas urbanas e rurais (CRUCIANI; MACHADO; SENTELHAS, 2002; BEIJO et al., 2003).

Como os projetos são feitos para o futuro, as suas demandas, seus benefícios e custos são todos conhecidos até certo limite. Dessa forma, erros na estimativa de valores hidrológicos podem acarretar prejuízos econômicos e ambientais (NERILO; MEDEIROS; CORDERO, 2002). O conhecimento das precipitações máximas pode-se evitar problemas como: erosão do solo, inundações, queda na produção agrícola, danificações de estradas, prejuízos em projetos de obras hidráulicas, danos em sistemas de drenagem, dentre muitas outras implicações (FREIRE et al., 2012).

Segundo Martins et al. (2011) geralmente os projetos hidráulicos são elaborados considerando o custo mínimo, associado a um risco admissível de falha, requer a previsão de grandezas hidrológicas de grande magnitude, tais como precipitações máximas e vazões máximas que podem vir a ocorrer em determinada região.

A precipitação máxima provável (PMP) é definida como a maior coluna pluviométrica correspondente a uma duração fisicamente possível de ocorrer sobre uma determinada área em uma dada época do ano e a disponibilidade de longas séries de precipitação, geralmente são mais frequentes do que as de vazão e que o estudo das precipitações máximas é um dos caminhos para se conhecer a vazão de enchente de uma bacia hidrográfica (TUCCI, 2001). O estudo de probabilidades fornece informações úteis sobre a chance de um determinado evento extremo ocorrer novamente em determinado espaço de tempo (FREIRE et al., 2013) e as séries de máximos valores são empregadas para ajuste, segundo a lei probabilística que melhor descreva o processo, possibilitando extrapolações (BEIJO; MUNIZ; NETO, 2005).

Vários autores têm utilizado a distribuição de Gumbel em estudos de precipitações máximas, sejam para determinar a precipitação máxima ou o período de recorrência de chuvas máximas ou ainda as curvas intensidade-duração-frequência. Estes trabalhos têm mostrado que a distribuição de probabilidade Gumbel é a que melhor se ajusta aos fenômenos meteorológicos, entre os quais a precipitação pluvial máxima. Beijo et al. (2003) estimaram a precipitação pluvial diária máxima esperada em diferentes níveis de probabilidade para a região de Jaboticabal (SP), ajustando a distribuição de Gumbel. Ferreira, Daniel e Tomazela (2005) confirmaram esse bom ajuste da distribuição Gumbel para dados de precipitação máxima de 165 localidades do Estado de São Paulo. (SANSIGOLO, 2008), ao comparar o ajustamento de diversas distribuições de probabilidade dos tipos Normal, Gumbel, Fréchet, Weibull, Log-Normal e Pearson, aos dados de precipitação máxima diária e de temperaturas máximas absolutas, coletados na cidade de Piracicaba, SP (19172006), mostrou que a distribuição Gumbel teve o melhor ajustamento enquanto Hartmann, Moala e Mendonça (2011) comprovaram um bom ajuste da distribuição Gumbel para os dados da precipitação máxima mensal para a região de Presidente Prudente.

O objetivo desse trabalho é determinar as precipitações máximas para diferentes períodos de retorno através da distribuição de Gumbel, para o município de Iguatu no Ceará.

\section{MATERIAL E MÉTODOS}

Para realização desse trabalho utilizou-se uma série de dados de precipitação coletados na cidade de Iguatu $(\mathrm{Fi}-$ gura 1 que está localizada nas coordenadas geográficas $6^{\circ} 22^{\prime} \mathrm{S}$ de latitude, $39^{\circ} 18^{\prime} \mathrm{W}$ de longitude e altitude 217,67 m, situa-se no centro-sul do estado do Ceará e possui características climáticas tipicamente tropicais. Segundo a classificação de Köppen o clima é BSw'h', semiárido com chuvas de outono e temperatura média mensal superior a $18^{\circ} \mathrm{C}$. A temperatura média anual é de 26 a $28^{\circ} \mathrm{C}$ (IPECE, 2004) com precipitação média anual de $983 \mathrm{~mm}$.

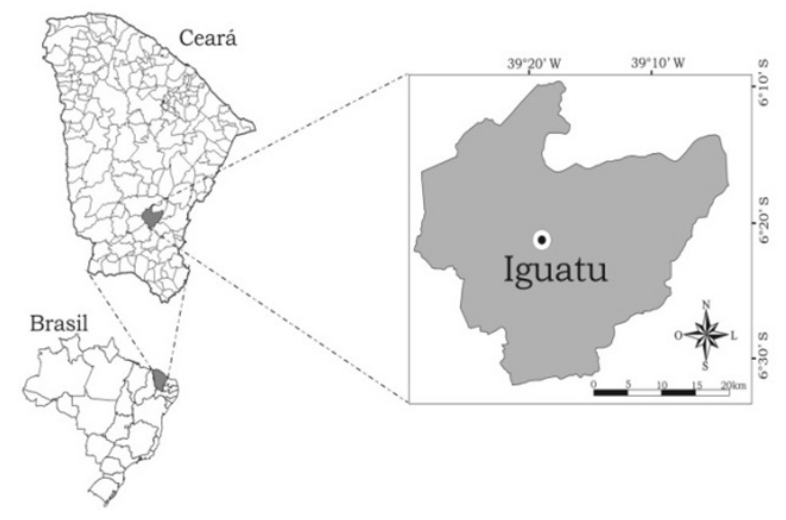

Figura 1: Localização da cidade em estudo.

O regime pluvial apresenta uma distribuição fortemente assimétrica a esquerda (Figura 2) e caracterizase por chuvas de alta intensidade e elevada variabilidade espacial e temporal.

Foram utilizados dados de precipitação pluviométrica máxima diária anual e máxima diária mensal anual, coletados de 1974 a 2012 (FUNCEME), 2013), expressas em altura de lâmina d'água $(\mathrm{mm})$, perfazendo 


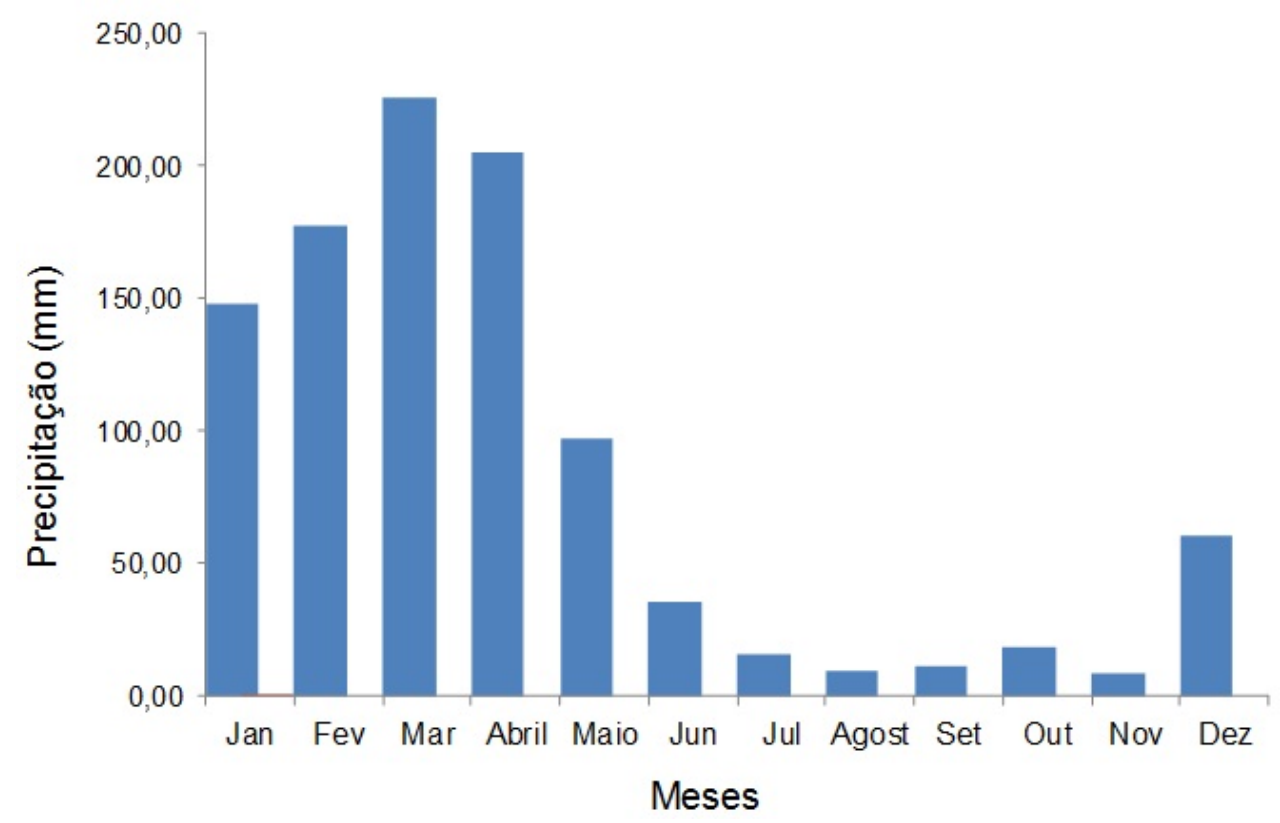

Figura 2: Precipitação média mensal do município de Iguatu, Ceará - série: 1974 a 2012 (FUNCEME. 2013).

um total de 38 anos, permanecendo dentro da faixa recomendada pela Organização Mundial de Meteorologia, que é, segundo Pinto (1999), de 30 anos.

Os dados foram organizados anualmente e posteriormente foram feitos os cálculos do período de retorno pela distribuição de Gumbel, através das séries de precipitação pluviométricas máximas utilizadas no período avaliado. Seus parâmetros foram encontrados por meio do método dos momentos. Sua Função Cumulativa de Probabilidade é representada pela Equação 1

$$
F C P: P(X \leq x)=\exp \left(-\exp \left(-\left(\frac{-x-\alpha}{-\beta}\right)\right)\right)
$$

Em que $\alpha$ é o parâmetro de escala e $\beta$ o parâmetro de forma; $x$ são os valores de precipitação máxima diária anual. Os parâmetros são encontrados pelas equações $2 \mathrm{e} 3$

$$
\begin{gathered}
\beta=\frac{\sqrt{6}}{\pi} S \\
\alpha=\bar{X}-0,5772 . \beta
\end{gathered}
$$

Em que $x$ é a média das precipitações e $S$ é o desvio padrão das precipitações.

O período de retorno é uma maneira de estimar, a partir de dados observados, a previsão de futuras ocorrências de certo evento.

O tempo de retorno é calculado utilizando-se a Equação 4 .

$$
T R=\frac{1}{1-P}
$$

Em que $P$ é a probabilidade do evento acontecer (adimensional). $T R$ é o tempo de retorno em anos.

A Equação 5 representa a estimativa da precipitação máxima em função dos parâmetros $\alpha$ e $\beta$, além do período de retorno.

$$
X=\beta-\operatorname{Ln}\left(-\operatorname{Ln}\left(1-\frac{1}{T R}\right)\right) . \alpha
$$

\section{RESULTADOS E DISCUSSÃO}

Observa-se que anualmente as chuvas diárias máximas mensais (acumuladas em 24 horas) da cidade de Iguatu são maiores do que 50 mm (Figura 3 e dos 39 anos analisados ocorreu em 15 anos uma chuva máxima maior do que $100 \mathrm{~mm}$, favorecendo para o aumento da estimativa da chuva máxima provável.

Na série destaca-se à chuva máxima diária ocorrida no dia 31 de janeiro de 1980, que foi de $174 \mathrm{~mm}$ provocou inundações em várias ruas de Iguatu. A cidade de Iguatu apresenta problemas de inundações por estar situada em uma região plana e com presença de lagoas, associado a uma desordenada urbanização que propicia a impermeabilização do solo diminuindo a infiltração, como também a deficiência dos canais de drenagem. A avaliação da precipitação permitirá prever a intensidade dos eventos e a frequência com que a população 


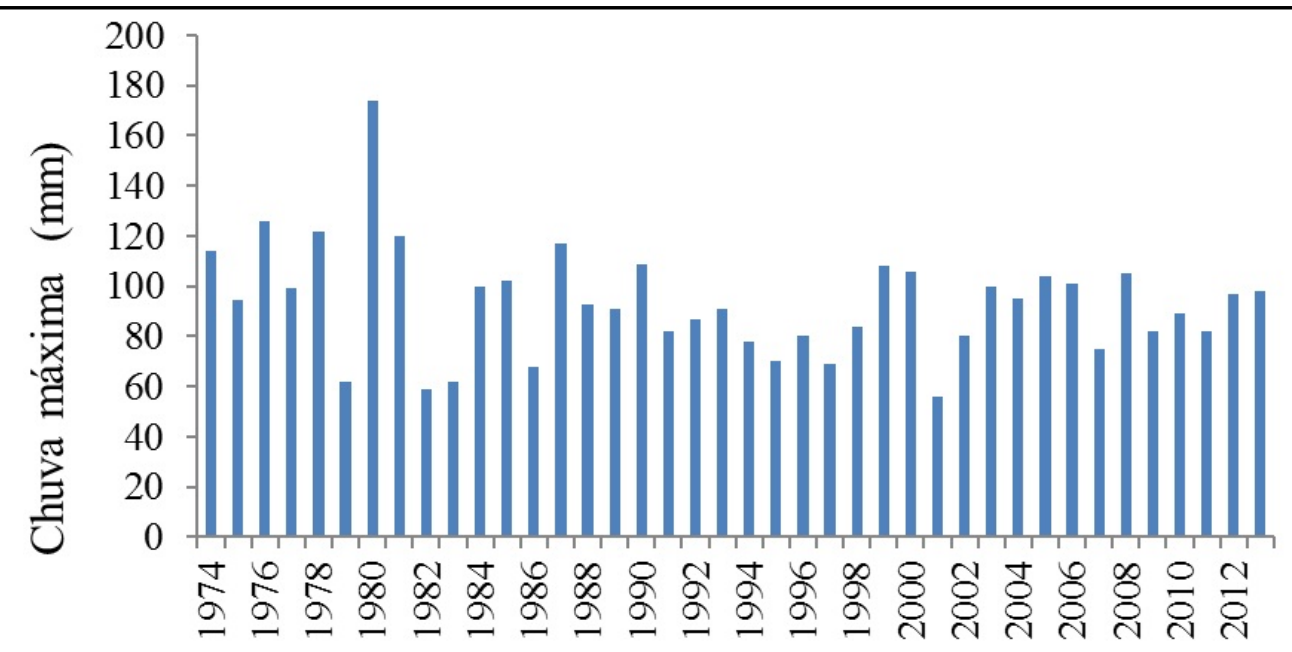

Anos

Figura 3: Representação gráfica da série de chuvas diárias máximas mensais (em mm) no município de Iguatu-CE, no período de janeiro de 1974 a dezembro de 2012 .

terá de enfrentar este problema. A escolha do período de retorno para obras hidráulicas é função dos prejuízos socioeconômicos com a ocorrência da precipitação máxima, os valores mostrados na tabela 1 indicam que ocorrerá inundação num período de retorno menor do que 5 anos.

$\mathrm{Na}$ Tabela 1 é apresentada as precipitações máximas para diversos períodos de retorno, extraídas da equação encontrada, através da aplicação do método de Gumbel.

Tabela 1: Precipitações máximas em função do período estabelecido

\begin{tabular}{cc}
\hline $\begin{array}{c}\text { Tempo de Retorno } \\
\text { (anos) }\end{array}$ & $\begin{array}{c}\text { Precipitação Máxima } \\
(\mathrm{mm})\end{array}$ \\
\hline 2 & 45,60 \\
5 & 140,17 \\
10 & 202,78 \\
20 & 262,84 \\
50 & 340,84 \\
100 & 398,84 \\
\hline
\end{tabular}

Nos resultados obtidos da precipitação máxima é possível visualizar, que probabilisticamente a cada 2 anos ocorre uma precipitação igual ou superior 45,60 $\mathrm{mm}$, sendo que esta mesma interpretação serve para os demais períodos de retorno. E as estimativas das precipitações pluviais máximas diárias aumentam à medida que se aumenta o tempo de retorno (Tabela 1). A probabilidade de ocorrer uma chuva igual ou superior a $398,84 \mathrm{~mm}$ por dia no tempo de retorno de 100 anos é de $1 \%$, enquanto uma chuva de $45,60 \mathrm{~mm}$ tem a pro- babilidade de $50 \%$ de ser igualada ou superada a cada 2 anos.

Estudo realizado por Freire et al. (2012) de uma série de 38 anos da precipitação máxima de um dia para os períodos de retorno de 2 e 100 anos da cidade de Mossoró, utilizando o método de Gumbel, encontrou para o tempo de retorno de 2 anos uma precipitação máxima 46,57 mm, valor muito aproximado para o mesmo tempo de retorno de Iguatu, o mesmo não ocorrendo para o tempo de retorno de 100 anos $(303,78 \mathrm{~mm})$ que diferiu em 95,06 $\mathrm{mm}$, provavelmente em virtude da precipitação média máxima diária anual de Iguatu ser superior a de Mossoró-RN. Freire et al. (2013) estimaram a precipitação máxima diária para a cidade de São João do Juaguaribe-CE valores muito próximos aos encontrados na cidade de Mossoró-RN, de 41,71mm e $304,91 \mathrm{~mm}$ respectivamente para os tempos de retorno de 2 e 100 anos.

As chuvas máximas tende a estabilizar com o aumento do período de retorno, apresentando o gráfico de sua curva um ajuste do comportamento logaritmo $\mathrm{Fi-}$ gura 4.

A Figura 4 mostra que o método de Gumbel mostrou-se adequado na estimativa das chuvas máximas para a cidade de Iguatu e confirmam as observação de Freire et al. (2012) e de Nerilo, Medeiros e Cordero (2002) de que é uma das mais utilizada no Brasil e no mundo no ajuste de eventos meteorológicos extremos.

Nos cinco primeiros meses do ano, as precipitações máximas mensais superam os outros meses do ano em 
ESTUDOS DAS CHUVAS MÁXIMAS PARA O MUNICÍPIO DE IGUATU-CE

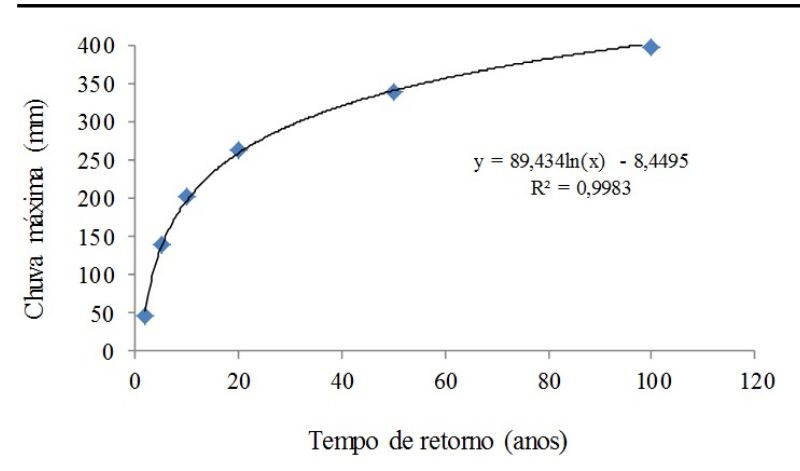

Figura 4: Chuvas máximas em função do tempo de retorno.

todos os períodos de retorno, com destaque para o mês de janeiro como o mês de maiores chuvas máxima em todos os tempos de retorno (Tabela 2.

É possível observar, a cada 2 anos, uma precipitação igual ou superior a 41,12 mm no mês de janeiro, sendo que esta mesma interpretação serve para os demais períodos de retorno e meses do ano. Os meses mais secos que vão de julho a novembro apresentaram os menores valores de chuva máxima, com destaque para o mês de novembro, que apresentou os menores valores de chuvas máximas para todos os períodos de retorno (Tabela 2).

Estudo desenvolvido por Araújo (2006) na bacia hidrográfica do rio Paraíba identificou que as chuvas máximas se concentram predominantemente nos meses de janeiro a abril, isso demonstra que a distribuição das chuvas máximas na bacia do rio Paraíba e se assemelha com a de Iguatu. Santos et al. (2014), ao avaliar as precipitações máximas para o município de Mossoró-RN, obteve o valor mínimo da precipitação máxima ocorreu no mês de setembro e o máximo no mês de fevereiro.

\section{CONCLUSÕES}

A precipitação máxima varia de $45,60 \mathrm{~mm} \mathrm{dia}^{-1}$ a $398,84 \mathrm{~mm} \mathrm{dia}^{-1}$ para os diferentes períodos de retorno em estudo. Os meses de novembro e dezembro são os que apresentam menores e maiores valores de chuvas máximas respectivamente. O método de Gumbel mostrou-se adequado na estimativa das chuvas máximas para a cidade de Iguatu.

\section{REFERÊNCIAS}

ARAÚJO, E. L. d. Análise estatística de chuvas intensas na bacia hidrográfica do rio Paraíba. Dissertação (Mestrado) - Universidade Federal de Campina Grande, Campina Grande, 2006. 94f.
BEIJO, L. A.; MUNIZ, J. A.; NETO, P. C. Tempo de retorno das precipitações máximas em lavras $(\mathrm{mg})$ pela distribuição de valores extremos do tipo i. Ciência e Agrotecnologia, v. 29, n. 3, p. 657 - 667, 2005.

BEIJO, L. A.; MUNIZ, J. A.; VOLPE, C. A.; PEREIRA, G. T. Estudo da precipitação máxima em jaboticabal, sp, pela distribuição de gumbel utilizando dois métodos de estimação dos parâmetros. Revista Brasileira de Agrometeorologia, v. 11, n. 1, p. 141 147, 2003.

CRUCIANI, D. E.; MACHADO, R. E.; SENTELHAS, P. C. Modelos da distribuição temporal de chuvas intensas em piracicaba, sp. Revista Brasileira de Engenharia Agrícola e Ambiental, v. 6, n. 1, p. 76 - 82, 2002.

\section{FERREIRA, J.; DANIEL, L.; TOMAZELA, M.}

Parâmetros para equações mensais de estimativas de precipitação de intensidade máxima para o estado de são paulo - fase i. Ciência e Agrotecnologia, v. 29, n. 6, p. $1981-1829,2005$.

FREIRE, F. G. C.; BARRETO, H. B. F.; BATISTA, R. O.; SANTOS, W. d. O. Estudo das precipitações para diferentes períodos de retorno no município de são joão do jaguaribe/ce. Revista Brasileira de Agricultura Irrigada, v. 7, n. 5, p. $300-305,2013$.

FREIRE, F. G. C. et al. Estudo das precipitações máximas para o município de mossoró-rn, brasil. Revista Brasileira de Agricultura Irrigada, v. 6, n. 1, p. $3-7,2012$.

FUNCEME. Fundação Cearense de Meteorologia e Recursos Hídricos. Produtos e Serviços. Download de Séries Históricas. 2013. Acesso em: 18 dez. 2013. Disponível em: <http://.funceme.br/index.php/areas/ tempo/download-de-series-historicas $>$

HARTMANN, M.; MOALA, F. A.; MENDONçA, M. A. Estudo das precipitações máximas anuais em presidente prudente. Revista Brasileira de Meteorologia, v. 26, n. 4, p. 561 - 568, 2011.

IPECE. Instituto de Pesquisa e Estratégia Econômica do Ceará. Perfil básico municipal. Iguatu, 2004.

MARCUZZO, F. F. N.; GOULARTE, E. R. P. Índice de anomalia de chuvas do estado do tocantins. Geoambiente, online, n. 19, p. 55 - 71, 2012.

MARTINS, C. A. d. S.; ULIANA, E. M.; MORGAN, E.; REIS, E. F. d. Estimativa da vazão e da precipitação máxima utilizando modelos probabilísticos na bacia 
Tabela 2: Precipitações máximas em função do período estabelecido.

\begin{tabular}{ccccccc}
\hline \multirow{2}{*}{ Meses } & 2 & 5 & 10 & 20 & 50 & 100 \\
\hline Janeiro & 41,12 & 85,22 & 114,42 & 142,42 & 178,68 & 205,84 \\
Fevereiro & 35,89 & 79,99 & 109,19 & 137,2 & 173,45 & 200,62 \\
Março & 35,12 & 79,22 & 108,42 & 136,42 & 172,68 & 199,84 \\
Abril & 37,73 & 81,83 & 11,03 & 139,04 & 175,29 & 202,46 \\
Maio & 29,22 & 58,16 & 77,33 & 95,71 & 119,5 & 137,32 \\
Junho & 17,87 & 27,91 & 34,57 & 40,95 & 49,21 & 55,4 \\
Julho & 16,79 & 17,48 & 17,93 & 18,37 & 18,94 & 19,36 \\
Agosto & 12,23 & 13,15 & 13,77 & 14,36 & 15,12 & 15,69 \\
Setembro & 13,28 & 14,8 & 15,81 & 16,77 & 18,02 & 18,95 \\
Outubro & 21,09 & 25,65 & 28,67 & 31,57 & 35,32 & 38,13 \\
Novembro & 10,47 & 12,7 & 14,18 & 15,59 & 17,43 & 18,8 \\
Dezembro & 28,62 & 45,23 & 56,22 & 66,77 & 80,42 & 90,65 \\
\hline
\end{tabular}

hidrográfica do rio benevente. Enciclopédia Biosfera, v. 7, n. 13, p. 1130 - 1142, 2011. Centro Científico Conhecer - Goiânia.

NERILO, N.; MEDEIROS, P. A.; CORDERO, A.

"Chuvas intensas no estado de Santa Catarina". Santa Catarina, 2002. 156p.

PINTO, F. R. L. Equações de intensidades-duraçãofreqüencia da precipitação para os estados do Rio de Janeiro e Espirito Santo: estimativa e espacialização. Dissertação (Mestrado em Engenharia Agrícola) Universidade Federal de Viçosa, Viçosa, 1999. $70 \mathrm{f}$.

SANSIGOLO, C. Distribuições de extremos de precipitação diária, temperatura máxima e mínima e velocidade do vento em piracicaba, sp (1917-2006). Revista Brasileira de Meteorologia, v. 23, n. 3, p. 341-346, 2008.

SANTOS, W. d. O.; MESQUITA, F. d. O.; BATISTA, B. d. O.; BATISTA, R. O.; ALVES, A. d. S.

Precipitações máximas para o município de mossoró de 1964 a 2011 pela distribuição de gumbel. Irriga, v. 19, n. 2, p. 207-213, 2014.

TUCCI, C. Hidrologia - Ciência e Aplicação. Porto Alegre, 2001. 943p.

VIEIRA, J. P. G.; DE, S. M. J. H.; TEXEIRA, J. M.; CARVALHO, F. P. d. Estudo da precipitação mensal durante a estação chuvosa em diamantina, minas gerais. Revista Brasileira de Engenharia Agrícola e Ambiental, v. 14, n. 7, p. 762-767, 2010. 